\title{
Chapter 2 \\ Background: Laser Technology \\ and Applications to Clinical Surgery
}

The goal of this chapter is to provide the background that frames the research described in this dissertation. Principles and concepts relevant to the content of subsequent chapters are introduced here. Because this work resides at the intersection of laser-tissue interaction with computer-assisted surgery, the basics of laser technology are introduced here.

Modern surgical laser systems are advanced instruments whose functioning is based on technology derived from a variety of different disciplines, including optics, electromagnetism, electronics. Rather than describing the composition of a modern laser system in detail, here we present a generic description of the mechanisms that permit the generation of laser light. In laser surgery, laser beams are primarily used as scalpels, i.e. as tools to perform incisions and resections. Laser-induced effects on tissue are manifold; although some of them are advantageous, others can produce irreparable damage and diminish the overall quality of surgery. Here, we review these effects and explain how they can be generated and controlled.

The chapter starts with an overview of the physical phenomena that are exploited in the generation of laser light. An understanding of these phenomena is an essential prerequisite to understand the variety of laser sources available today: these are classified primarily by their wavelength, intensity and spot size. The properties of laser light will be described and contrasted with the characteristics of light produced by traditional incandescent sources. Laser sources produce highly intense beams of nearly monochromatic light, which are known to produce numerous interesting effects on the material they traverse. These effects will be discussed in the latter part of the chapter, which focuses on light-matter interactions. A class of interactions will be reviewed in greater detail, namely thermal interactions: these are implemented in numerous medical applications for therapeutic purposes. In the context of laser surgery, thermal interactions are used to produce incisions on tissue. The physical processes that enable the creation of laser incisions on biological tissue will be described. Finally, the advantages and shortcomings of laser cutting of tissues will be addressed. 


\subsection{Physical Properties of Light}

Our understanding of the nature of light is based on the fact that it exhibits properties of both electromagnetic (EM) waves and elementary particles, this characteristic is known as the wave-particle duality [1]. First, we consider the description of light as EM waves; the terms light and EM radiation are used interchangeably throughout this dissertation. Light can be regarded as the perturbation produced by the interplay of mutually related electric and magnetic fields. Each pair of electric/magnetic fields is characterized by a common wavelength $\lambda$ and oscillate perpendicular with each other and at right angle to the direction of propagation, as shown in Fig. 2.1. In general, EM radiation is classified according to the range of wavelengths it contains. The EM spectrum covers all the possible wavelengths of EM radiation: a limited interval is shown in Fig. 2.2, ranging from longer wavelengths (Microwaves) to shorter (XRays). A fundamental physical property of EM waves is the frequency $v$, which relates to the wavelength through the relation $c=v \cdot \lambda$, where $c$ is the speed of EM waves in vacuum.

The classic picture of light as EM waves can be revisited in the perspective of the quantum theory. In its simplest form, the theory describes light as the

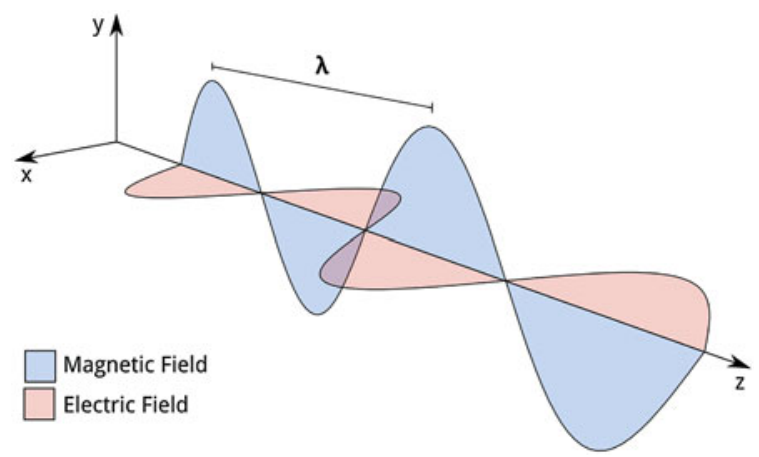

Fig. 2.1 Electromagnetic wave propagating along the $z$ axis of a Cartesian reference frame Oxyz. This wave is formed by a pair of mutually related electric and magnetic field, oscillating perpendicular to each other at the same wavelength $\lambda$

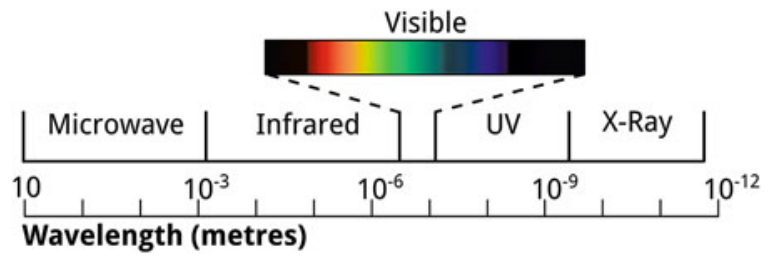

Fig. 2.2 Electromagnetic Spectrum. Note that the relation between wavelength $(\lambda)$ and frequency ( $v$ ) of an electromagnetic wave is $\lambda v=c$, where $\mathrm{c}$ is the speed of propagation of electromagnetic waves in vacuum 
Table 2.1 Radiometric quantities and units used throughout this dissertation

\begin{tabular}{l|l|l}
\hline Quantity & Symbol & Unit \\
\hline Power density, intensity, irradiance & $I$ & $\mathrm{~W} \cdot \mathrm{m}^{-2}$ \\
\hline Energy density, fluence & $E$ & $\mathrm{~J} \cdot \mathrm{m}^{-2}$ \\
\hline Volumetric power density & $S$ & $\mathrm{~W} \cdot \mathrm{m}^{-3}$ \\
\hline Volumetric energy density & $E_{v}$ & $\mathrm{~J} \cdot \mathrm{m}^{-3}$ \\
\hline
\end{tabular}

transport of energy through a region of space. The transport is associated with the flow of subatomic particles, called photons, each carrying an energy quantified by $E_{\text {photon }}=h v[1]$, where $v$ is the frequency of the electromagnetic wave, as per the EM wave description; $h$ is the Planck's constant.

As we shall see later in this chapter, energy created by a light source can be delivered to matter to alter its physical properties. The interaction of light with matter depends on the amount of energy involved, as well as on the size of the area (or volume) under irradiation. These quantities are often combined into a single radiometric parameter. Different parameters exist, their nomenclature is not uniform throughout the literature. In this dissertation the power density $I$, the energy density $E$, the volumetric power density $S$ and the correspondent energy density $E_{v}$ are most used. For the sake of clarity, Table 2.1 lists these quantities with their units and alternative names they are commonly attributed in the literature.

\subsection{Fundamentals of Lasers}

Laser stands as an acronym for Light Amplification by Stimulated Emission of Radiation. This term identifies the principle of operation of a class of devices that use optical amplification to produce an intense beam of highly directional, monochromatic light [2]. Stimulated emission is a physical process that occurs when a medium is exposed to an EM field: light is emitted as a result of the interaction of the field with the atoms of that medium. The emission is characterized by the same wavelength and phase of the incoming radiation. This contrasts with the other known emission process, i.e. spontaneous emission: in this case, light propagates in all directions with random wavelength and phase [2]. Spontaneous and stimulated emission are general phenomena that occur naturally at any light source: the intensity of each emission can be characterized by the number of photons that are released. It can be shown that, for a given medium in thermal equilibrium at temperature $T$, subject to an EM field at frequency $v$, the ratio of the photons emitted by spontaneous $\left(P_{s p}\right)$ and stimulated emissions $\left(P_{s t}\right)$ is

$$
\frac{P_{s p}}{P_{s t}}=\exp \left(\frac{h v}{k T}\right)-1,
$$

where $k$ is the Boltzmann's constant. This relation establishes the dependency of the light emission with the frequency of the EM field. At frequencies $v>k T / h$, spontaneous emission dominates the process: for instance, let us consider the 
Fig. 2.3 Basic structure of a laser system

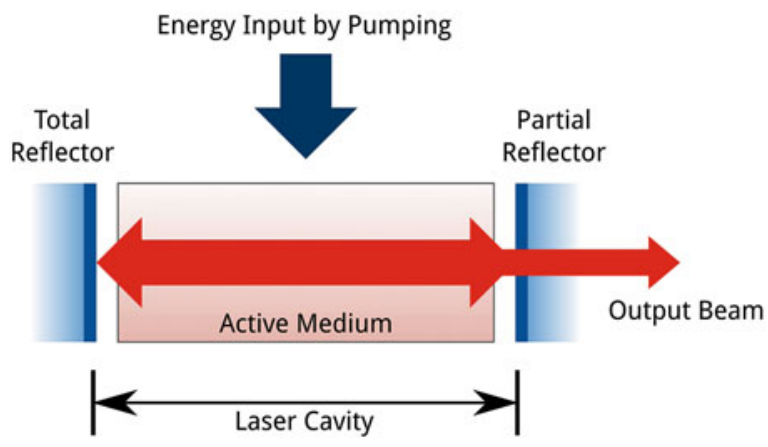

filament of a light bulb emitting yellow light $\left(v=5 \times 10^{14} \mathrm{~Hz}\right.$, see Fig. 2.2) at a temperature of $T=2000 \mathrm{~K}$, then Eq. 2.1 yields that $P_{s p} / P_{s t} \approx 1.5 \times 10^{5}$. This example is indicative of how the emission of visible traditional light sources consists predominantly of spontaneous emission. Conversely, stimulated emission strongly prevails at long wavelengths, e.g. in the far infrared and microwave ranges. To have significant stimulated emission at wavelengths shorter than far infrared, it is necessary that a particular condition is met, referred to as population inversion [2]. This entails altering the distribution of the atomic energy levels in a medium, so that more atoms reside in higher energy states than in the lower. A population inversion can be artificially created pumping energy into the medium, either by means of a traditional powerful light source (optical pumping) or establishing a current flow into it (electrical pumping) [2].

The basic working principle of a laser device is illustrated in Fig. 2.3. A laser system is typically composed of two main parts: (i) a cavity filled with an active medium and (ii) a pump source that injects energy into the medium, thus creating a population inversion that triggers the stimulated emission of light. The cavity is enclosed by two reflective optical elements which force the light to travel back and forth through the medium. Further stimulated emission is produced at each pass, resulting in an optical amplification effect. Once the system has built up a sufficient amount of energy, a fractional part of the light trapped in the cavity escapes through one the reflecting mirrors; this is designed in such a way to have non vanishing transmission at the laser characteristic frequency. The light that escapes the cavity forms the output beam of the laser. Several different materials can be employed as active medium: these include solids, gases, fluids, semiconductors; the choice of the medium determines the wavelength at which stimulated emission occurs.

\subsubsection{Laser Beam Optics}

The optical properties of a laser beam describe how this propagates through space and the light intensity associated with it. These properties are dictated by the design 
of the laser device; specifically by the geometry of the cavity and the shape of the mirrors used to confine light into it.

In general, the propagation of light within an homogeneous medium can be formulated in terms of a wave equation, i.e. a second-order partial differential equation that describes how the electric field $\mathbf{E}$ and the magnetic field $\mathbf{B}$ associated with the light wave behave in such material. The wave equation is derived from the Maxwell's equations: assuming a medium with relative permeability $\mu_{r}\left(N \cdot A^{-2}\right)$ and permittivity $\varepsilon_{r}\left(F \cdot \mathrm{m}^{-1}\right)$, the wave equation takes the form [3]:

$$
\begin{aligned}
& \nabla^{2} \mathbf{E}=\varepsilon_{0} \mu_{0} \varepsilon_{r} \mu_{r} \frac{\partial^{2} \mathbf{E}}{\partial t^{2}}, \\
& \nabla^{2} \mathbf{B}=\varepsilon_{0} \mu_{0} \varepsilon_{r} \mu_{r} \frac{\partial^{2} \mathbf{B}}{\partial t^{2}},
\end{aligned}
$$

where $\mu_{0}$ and $\varepsilon_{0}$ are the permeability and the permittivity of vacuum, respectively. Equation 2.2 can be solved to obtain a description of the propagation of light which escapes the cavity of a laser device. The form of the solution depends on the specific boundary conditions imposed by the size of the cavity, the shape and type of the reflective optical elements.

Gaussian Beams constitute a set of solutions to Eq. 2.2 that are commonly builtin in laser devices [3]. The simplest form of these solutions is associated with a Gaussian-shaped intensity profile, whose peak lies on the optical axis. This is commonly referred to as the fundamental Transverse Electromagnetic Mode (TEM), and is indicated by the designation $\mathrm{TEM}_{00}$. A more general form for the intensity profile can be calculated applying specific boundary conditions. Here we consider the output beam of a laser device with cylindrical symmetric cavity. These beams are described through the combination of a Gaussian function with a generalized Laguerre Polynomial of order $p$ and index $l$, i.e. $L_{p}^{l}$. Assuming a cylindrical reference frame $(r, \varphi, z)$, with $z$ denoting the beam axis, $r$ and $\varphi$ being the polar coordinates in a plane transverse to $z$, the optical power density is given by [4]:

$$
I_{p l}(r, \varphi, z)=I_{0} \rho^{l}\left[L_{p}^{l}(\rho)\right]^{2} \cos ^{2}(l \varphi) \exp (-\rho)
$$

where $\rho=2 \cdot r^{2} / w^{2}(z)$. The quantity $w(z)$ is called the spot size of the Gaussian Beam, defined as the radius at which the intensity of the $\mathrm{TEM}_{00}$ mode is $1 / e^{2}$ of its peak value $I_{0}$. This set of solutions is commonly referred to as $\operatorname{TEM}_{l p}$. The order and the index of the Laguerre polynomial determine the shape of the intensity profile, as shown in Fig. 2.4.

$\mathrm{TEM}_{00}$ beams present several interesting properties. The intensity profile of these beams maintain a Gaussian shape regardless of the selection of the cross section along the propagation axis $z$. Furthermore, these beams present a relatively low divergence; this is a measure of how large the variation of the spot size $w(z)$ is along $z$. This concept is illustrated in Fig. 2.5, where the $w(z)$ is plotted. The spot size $w(z)$ of the beam follows an hyperbolic law, and presents a global minimum $w_{0}$ where 


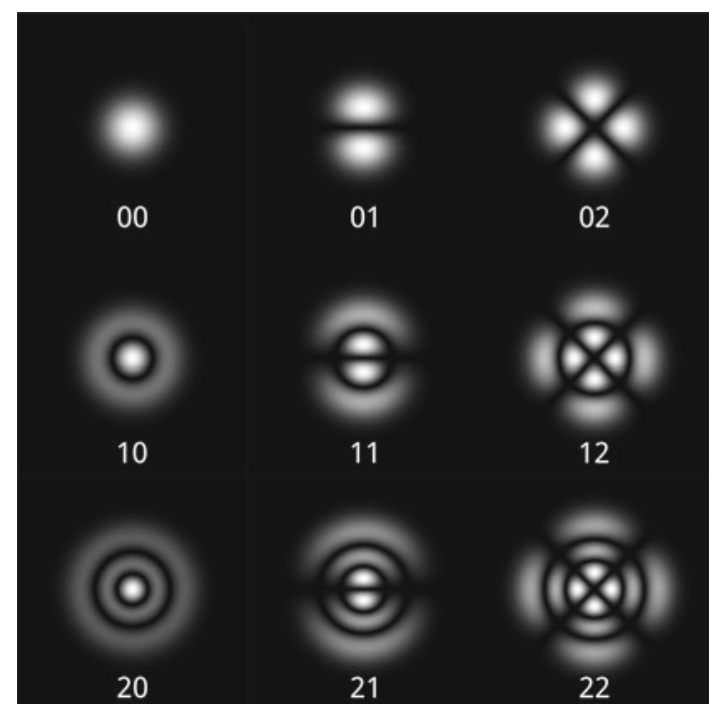

Fig. 2.4 Transverse modes of Gaussian Beams for different values of $l$ and $p$. Higher values of intensity are represented with brighter shades of gray

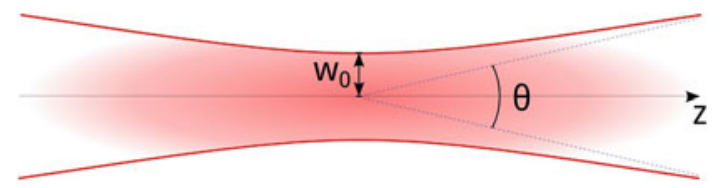

Fig. 2.5 Propagation of a TEM 00 beam along an optical axis $z$. The divergence angle $\theta$ is shown, which is related to the variation of the spot size of the beam $w(z)$. The spot size measured at the maximum focusing point is denoted with $w_{0}$ and is called the beam waist

the focusing reaches its maximum. This quantity is known as the beam waist. The divergence angle $\theta$ is defined in terms of $w_{0}$ and the wavelength $\lambda$, i.e.,

$$
\theta=\frac{2 \lambda}{\pi w_{0}} .
$$

From this relation it follows that the divergence increases linearly with the wavelength of the laser beam. Also, the divergence of a beam is inversely proportional to the beam waist $w_{0}$. It should be noted that collimated beams of light could be produced also through incoherent light sources. However, this requires the use of a focusing optical system that would greatly attenuate the energy density of the output. In contrast, the collimation of a laser beam does not entail significant losses of optical power, thus permitting the creation of large energy densities even at low output power. 
Fig. 2.6 Exemplary laser emission spectrum, centered at $\nu_{0}$ and with Frequency Spread $\Delta v$

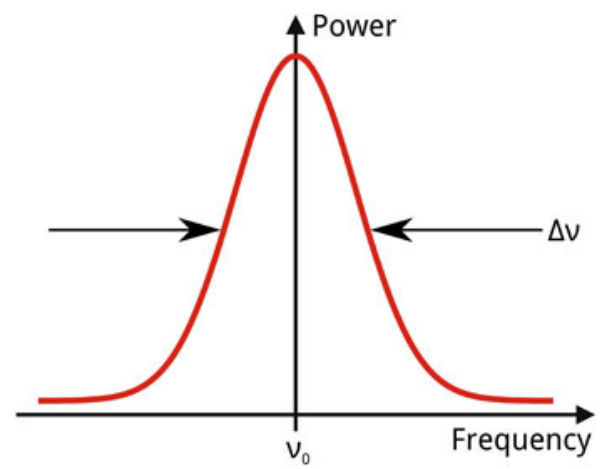

\subsubsection{Spectral Properties of Laser Light}

The light generated by a laser device is coherent, i.e. the EM waves that form a laser beam bear a fixed phase relationship to each other, thus presenting a high degree of correlation. Laser radiation presents both spatial and temporal coherence. The former refers to the property that the phase difference measured between any pair of points on the wavefront is uniform. Similarly, temporal coherence indicates that the phase of a wave remains constant through time. The coherence of laser light is a direct result of the stimulated emission process, as the emitted photons present the same phase, frequency and direction of stimulating photons [2].

It can be shown that high temporal coherence entails a narrow emission spectrum [2] - this explains why laser devices appear to produce nearly monochromatic light. Typical values of frequency spread $\Delta v$ (Fig. 2.6) for a laser range from $1 \mathrm{GHz}$ down to a few Hertz, depending on the properties of the active medium and the design of the cavity. Laser devices are commonly described by the wavelength $\lambda$ associated with the center $v_{0}$ of their emission spectrum. For instance, most medical carbon dioxide $\left(\mathrm{CO}_{2}\right)$ lasers present an emission in the mid-infrared range, centered at 10.6 $\mu \mathrm{m}[5]$.

\subsection{Fundamentals of Laser-Matter Interaction}

Because of its unique properties, laser radiation interacts with matter differently with respect to the light emitted by a traditional incoherent light source. Laser-matter interaction is an active area of research, which aims to investigate and understand the manifold physical phenomena that occur when matter is exposed to laser light. Most of these phenomena have been extensively studied and reviewed in numerous scholarly works [5-7].

Mechanisms of laser-matter interaction have been classified in various ways in the literature. Here, we start our discussion presenting general concepts related to 
Fig. 2.7 Basic interactions of laser light with matter. Here, the beam is directed onto the surface of a block of material. Depending on the properties of the beam and the material, three different interaction mechanisms may occur: (a) Transmission (b) Attenuation (c) Reflection and Refraction
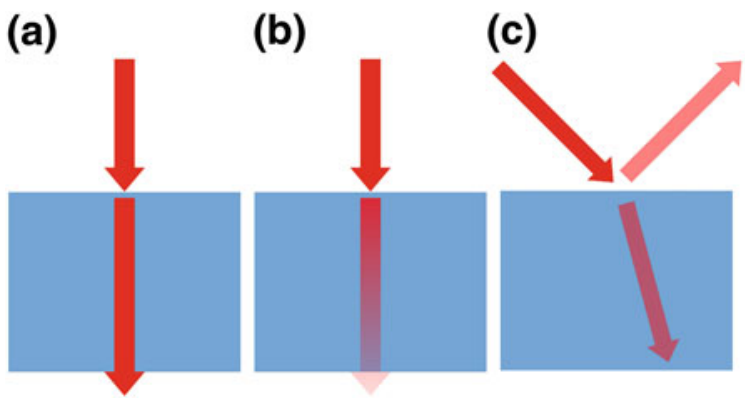

how matter affects the propagation of a laser beam. When laser light is incident on a material, three basic effects can be observed, as illustrated in Fig. 2.7:

- Transmission refers to the undisturbed propagation of light through the material. The material is said to be transparent: light travels through it without any attenuation, maintaining the same direction of propagation. The energy density entering the medium equals the one that escapes it.

- Attenuation occurs when the energy associated with the laser beam is lost within the material volume. The propagation of the beam through the material results in an attenuation of its energy density, either because energy is absorbed by the material or it is dispersed into it. Basic attenuation mechanisms are absorption and scattering [5].

- Reflection and Refraction occur when light crosses the boundary between materials with different optical properties. A fraction of the incident wave is returned from the surface of the material (reflection), while the remaining part propagates into it (refraction). Refraction is usually associated with a change in the speed and direction of propagation. Reflection and refraction are strongly related to each other by the Fresnel's Equations [8].

In general, these effects might occur simultaneously in a combined fashion, i.e. when light impinges on the surface of a material part of it is reflected, part is transmitted and part is either absorbed or dispersed into the material. The proportions of light which is transmitted, returned or dispersed are dictated by the incident wavelength and the optical properties of the material. The principle of energy conservation holds: the sum of the energies associated with each of these interactions gives the total amount of incident energy.

In the scope of this doctoral thesis, we shall focus on a specific set of laser-matter interactions, in which the target material consists of biological tissue. Most relevant laser-tissue interactions are induced by the absorption of laser energy within the tissue volume. Among these, thermal interactions play a crucial role in laser surgery, as we will find later on. In the next section, we present the fundamentals of lasertissue interaction mechanisms, with particular focus on the effects that laser radiation can produce on tissues. 


\subsection{Interactions of Lasers with Biological Tissues}

Biological tissues are optically turbid materials: absorption and scattering dominate the interactions of these media, thus enabling an energy transfer process from the laser source to the tissue [5]. The capability of laser light to penetrate a tissue and deposit energy within its volume is the key of numerous therapeutic applications: lasers can be employed to precisely deliver an energy dose to a selected target volume within an organ, e.g. to destroy malignant tissue. In these applications, the spatial distribution of energy induced by the laser is essential for the purpose of predicting successful treatment. Such distribution is described by the volumetric energy density $E_{v}\left(\mathrm{~J} \cdot \mathrm{m}^{-3}\right)$, and depends on the incident wavelength as well as on the optical properties of the target [5,9].

Relevant optical properties of tissues include the coefficients of absorption $\mu_{a}$ $\left(\mathrm{m}^{-1}\right)$ and scattering $\mu_{s}\left(\mathrm{~m}^{-1}\right)$. The former determines the fraction of incident energy absorbed by the tissue; the latter is related to how deep laser radiation penetrates into the tissue. Both coefficients are determined by the molecular composition of the target. Because different molecules respond selectively to different wavelengths, these coefficients are wavelength-dependent [10]: for instance, Fig. 2.8 presents the absorption spectra of three different types of human tissues, namely skin, aortic wall and cornea. In the considered range of wavelengths, skin presents the largest absorption due to the presence of melanin molecules, which are known to strongly absorb radiation in the visible range (wavelength: 400-780 nm) [5, 9]. The absorption of aortic tissue is characterized by haemoglobin, which is also a strong absorber in this range. Conversely, cornea does not have any constituent that presents a significant absorbance, thus resulting almost transparent. In general, different types of tissues present different absorption and scattering coefficients. Numerous works

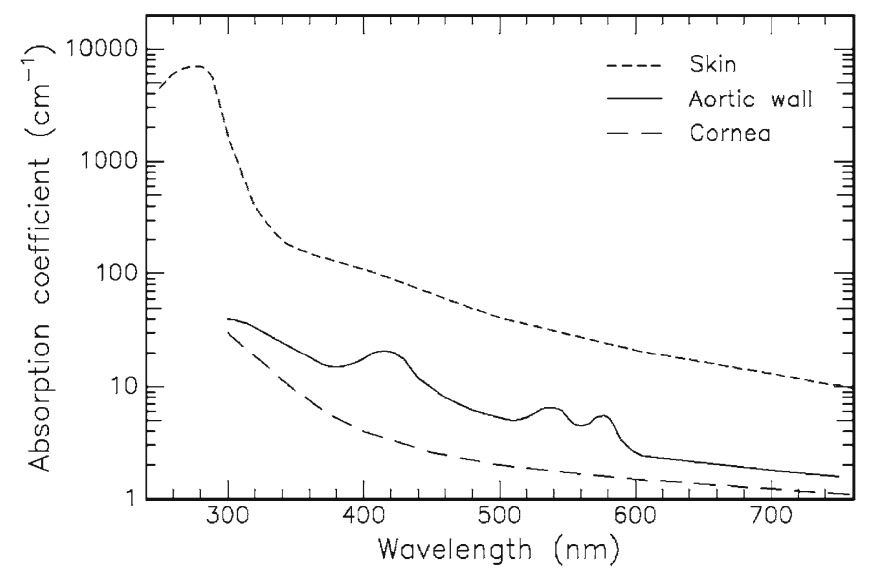

Fig. 2.8 Wavelength-dependency of the absorption coefficients of skin, aortic wall, and cornea. Reproduced from [5] with kind permission from Springer Science+Business Media 
report tabulated values of these coefficients sampled on various tissues and for different wavelengths [10]. Nonetheless, slight variations from the reported values may be observed in practice, due to the inherent inhomogeneity of biological tissues: these may present variations in blood content, water content, fiber development, etc. $[5,10]$.

Laser energy can act on tissues, inducing alterations at the physical and chemical levels. Diverse effects can be produced, depending on the optical properties of tissues discussed above, as well as on the properties that characterize the laser beam: wavelength, beam intensity and waist. Another important parameter is the total time of laser exposure: this is often used in conjunction with the beam intensity to classify laser-tissue interactions, as shown in Fig. 2.9. Five main interaction classes can be distinguished, namely photodisruption, plasma-induced ablation, photoablation, thermal interactions and photochemical interactions [5]. It is interesting to observe all these interactions occur for similar values of the applied energy density, i.e. between 1 and $1000 \mathrm{~J} \cdot \mathrm{cm}^{-2}$. This result shows the practical importance of the exposure time, which can be tuned to selectively enable a specific interaction [5].

For the purpose of this dissertation we shall focus on thermal interactions. For further details on the other types of interactions, the reader is referred to [5].

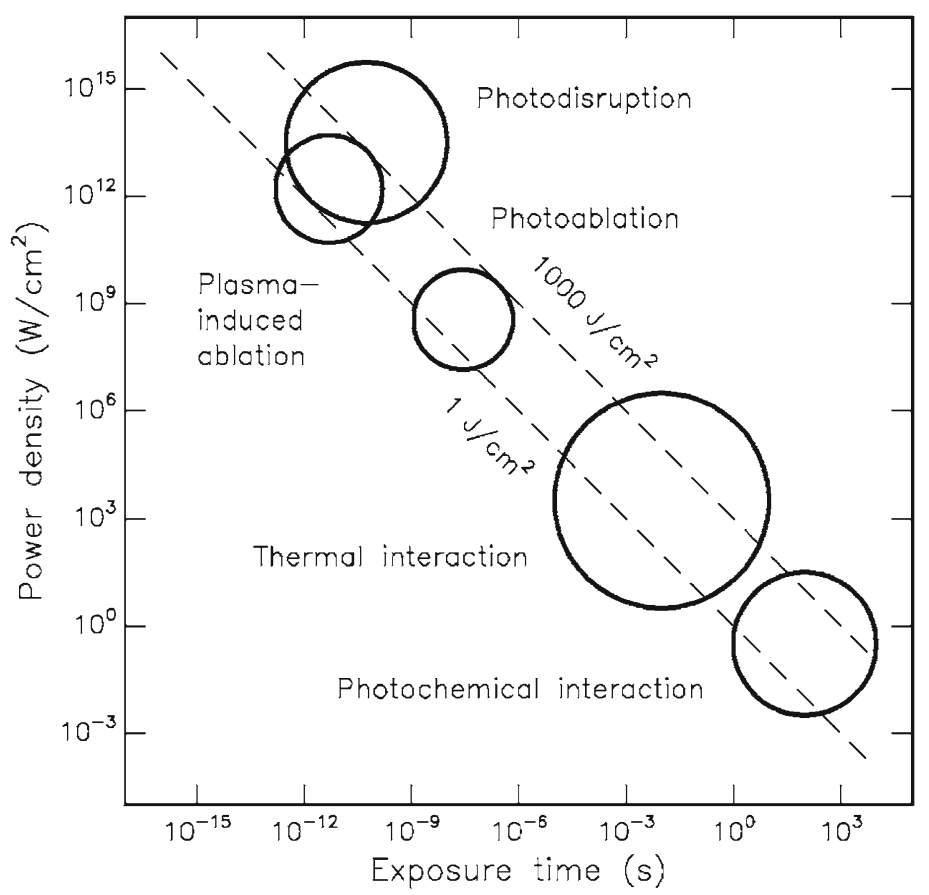

Fig. 2.9 Classification of thermal laser-tissue interactions types. Reproduced from [5] with kind permission from Springer Science+Business Media 


\subsubsection{Thermal Interactions}

Thermal interactions constitute a large group of interaction types, characterized by irradiations longer than $1 \mu \mathrm{s}$ and shorter than $1 \mathrm{~min}$. During these interactions, laser energy is absorbed by the tissue under the form of heat, thereby determining a local increase of temperature [5]. As we shall see later on, several different effects can be produced on tissue, depending on the duration and peak value of temperature achieved. These effects range from hyperthermia $\left(45^{\circ} \mathrm{C}\right)$ to vaporization $\left(100{ }^{\circ} \mathrm{C}\right)$ and carbonization $\left(>100{ }^{\circ} \mathrm{C}\right)$.

In the scope of laser surgery, laser-induced thermal effects are especially of importance. Vaporization is the process by means of which laser incisions are conducted: because soft tissues are largely composed by water, their molecules start to evaporate at approximately $100{ }^{\circ} \mathrm{C}$, determining a material removal (ablation) process. Although some laser-induced effects are advantageous, others are considered detrimental. It it the case of carbonization, that occurs at temperatures above $100{ }^{\circ} \mathrm{C}$. Carbonization determines the formation of scar tissue, thus diminishing the quality of the surgical procedure. It is important to note that temperature is the parameter that governs the onset of these effects. Therefore, for the purpose of understanding the diverse thermal effects that lasers can produce on tissues, we must establish a model for the spatial and temporal evolution of tissue temperature during laser irradiation. In the following paragraphs we derive the equations that govern the generation of heat within the tissue and see how the generation of heat is related to the variation of temperature. Then, we will review and discuss the diverse thermal effects that lasers can produce on tissues.

Let us consider a sample of homogeneous tissue exposed to a $\mathrm{TEM}_{00}$ laser beam, as depicted in Fig. 2.10. For the sake of simplicity, we assume that the surface of tissue exposed to the beam is plain and normally oriented with respect to it. A cylindrical reference frame $(r, \vartheta, z)$ is used to describe the tissue geometry, with $z$ being coincident with the beam axis and $r$ representing the radial distance with respect to such axis. Without any loss of generality, cylindrically symmetry is assumed. The

Fig. 2.10 Tissue geometry.

Adapted from [11]

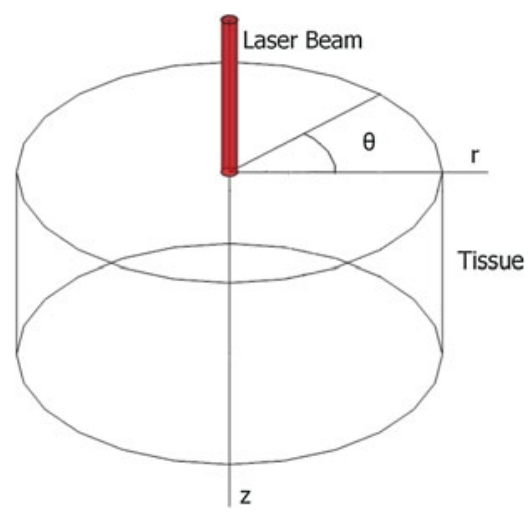


beam intensity inside the tissue is then described by Niemz [5]:

$$
I(r, z, t)=I_{0} \exp \left(-\frac{2 r^{2}}{\omega^{2}}-\mu_{a} z\right) \exp \left(\frac{-8 t^{2}}{\tau^{2}}\right),
$$

where $I_{0}$ is the beam intensity incident on the surface of tissue, $\mu_{a}$ is the coefficient of absorption of the tissue, $\omega$ is the beam waist, $t$ is the total exposure time and $\tau$ is the pulse duration. This model of intensity does not take into account the effect of scattering, which can be neglected for most practical applications on soft tissues [5]. One important parameter associated with the spatial distribution of intensity within the tissue is the absorption length, defined as the inverse of the absorption coefficient $\mu_{a}$ :

$$
L=\frac{1}{\mu_{a}}
$$

The absorption length measures the distance, along the beam axis $z$, at which the intensity has dropped to $1 / e$ of its incident value $I_{0}$ [5].

The rate of heat generation within the tissue $S(r, z, t)\left(\mathrm{W} \cdot \mathrm{m}^{-3}\right)$ is proportional to the intensity $I$, with the coefficient of absorption $\mu_{a}$ being the proportional factor [5]:

$$
S(r, z, t)=\mu_{a} I(r, z, t)
$$

Having identified a model for the rate of heat generation, we now focus on the equations that permit to quantify the consequent variation of temperature [5]. Assuming that neither phase transitions (e.g. vaporization) nor tissue alterations (e.g. coagulation) occur, a basic law of thermodynamics can be used to model the change of temperature $d T$ related to a variation of heat content $d Q$ :

$$
d T=\frac{d Q}{m c}
$$

where $m$ is the mass of tissue, and $c$ is its specific heat capacity, expressed in $\mathbf{J}$. $\mathrm{kg}^{-1} \cdot \mathrm{K}^{-1}$. A variation of heat content can occur either because of the energy deposited by the heat source (Eq. 2.7) or due to heat conduction, i.e. the variation of heat content per unit of volume $(\dot{q})$, which is the primary mechanism by which heat is transferred to tissue structures not reached by the laser radiation. Other heat losses as heat convection and heat radiation can be neglected in first approximation [5].

It can be shown, by means of the equation of continuity ${ }^{1}$ and the general diffusion equation, ${ }^{2}$ that the volumetric variation of heat $\dot{q}$ depends on the temperature by the following relation:

$$
\dot{q}=k \Delta T
$$

\footnotetext{
${ }^{1}$ The equation of continuity establishes the relation between the heat flow and the temporal change in heat content per unit of volume, $\dot{q}$.

${ }^{2}$ The general diffusion equation states that the heat flow is proportional to the temperature gradient.
} 
where $\Delta$ is the Laplace operator and $k$ is the tissue heat conductivity, expressed in $\mathrm{W} \cdot \mathrm{m} \cdot \mathrm{K}$. Combining Eq. 2.9 into Eq. 2.8 yields:

$$
\dot{T}=\frac{1}{m c} \dot{Q}=\frac{1}{\rho c} \frac{\dot{Q}}{V}=\frac{1}{\rho c} \dot{q}=\frac{1}{\rho c} k \Delta T
$$

where $V$ is the volume of the tissue $\left(\mathrm{m}^{3}\right), \rho$ is the tissue density $\left(\mathrm{kg} \cdot \mathrm{m}^{-3}\right)$ and $c$ is its specific heat capacity $\left(\mathrm{J} \cdot \mathrm{kg}^{-1} \mathrm{~K}^{-1}\right)$. Equation 2.10 can be extended to account for the heat generated by the laser:

$$
\dot{T}=\frac{1}{\rho c}(k \Delta T+S) .
$$

Using the Laplace operator for the cylindrical reference frame, we obtain:

$$
\dot{T}=\kappa\left(\frac{\partial^{2}}{\partial r^{2}}+\frac{\partial^{2}}{r \partial r}+\frac{\partial^{2}}{\partial z^{2}}\right) T+\frac{1}{\rho c} S .
$$

Here, $\kappa$ is the temperature conductivity of tissue, which relates both constants, the specific heat capacity $(k)$ and the heat conductivity $(c)$. Azimuth symmetry is evidenced in the expression above. Exact solution of this inhomogeneous differential equation is rather complex. It involves the use of several approximations and assumptions, as we shall see in greater detail in Chap. 4. Numerical methods are commonly used to solve the time-dependent function that describes the temperature $T(r, z, t)$.

The diffusion of heat within tissue during laser irradiation is described by the thermal penetration depth $d_{\text {therm }}[5]$ :

$$
d_{\text {therm }}=\sqrt{4 \kappa t} .
$$

Analogously to the optical absorption length (Eq. 2.6), this term quantifies the depth at which the temperature has dropped to $1 / e$ of the superficial value, i.e. with respect to $T(z=0)$. Equating the optical absorption length with the thermal penetration depth, we obtain the following time-dependent relation:

$$
\frac{1}{\mu_{a}}=\sqrt{4 \kappa t},
$$

whose solution $\tau_{\text {therm }}$ is called the thermal relaxation time. This quantity gives an indication of how quickly a tissue dissipates the heat generated by a laser. For laser exposures shorter than $\tau_{\text {therm }}$, we have that $L>d_{\text {therm }}$, i.e. the diffusion of heat is contained in the volume reached by the laser radiation. By contrast, exposure times longer than $\tau_{\text {therm }}$ determine a significant diffusion of heat also to surrounding tissues, which are not under direct laser irradiation. As we shall see in the following, this result has great practical relevance: it gives a criterion for the selection of the laser exposure time, based on the thermal effects one wants to achieve. 
Having established the equations that govern the thermal response of tissues subject to laser irradiation, we now focus on the different biological effects that a thermal interaction may produce. Depending on the duration and peak value of temperature achieved, different effects like coagulation, vaporization, carbonization, and melting may be distinguished. These effects are listed in Table 2.2, together with the temperature at which they occur. Elevating the temperature of tissue up to $60{ }^{\circ} \mathrm{C}$ does not induce any permanent alteration, given that this condition is maintained for a time duration in the range of seconds [5]. Above $60{ }^{\circ} \mathrm{C}$ [5], coagulation occurs. This is a form of irreversible tissue necrosis which causes cells to cease their function. A phase transition is induced at $100{ }^{\circ} \mathrm{C}$ : this is temperature at which the the free water molecules in the tissue start to vaporize. As the volume of water tends to increase during the transition, a pressure build-up occurs. Increasing pressure eventually leads to micro explosions that tear up the fabric of tissue and produce craters. In the literature, this process is commonly referred to as thermal decomposition [5]. Another effect that may occur during a thermal interaction is carbonization. This occurs when the tissue temperature rises above $100{ }^{\circ} \mathrm{C}$, i.e. when laser irradiation continues after the water content of tissue has been completely evaporated.

Medical applications of lasers typically aim to induce one specific thermal effect on tissue. Vaporization is exploited in laser surgery to create ablations and produce incisions on tissue $[5,12,13]$. In practice, several thermal effects might occur simultaneously, depending on the parameters that characterize the laser irradiation. The locations and extent of each effect follows the spatial distribution of temperature produced during and after the laser exposure [5]. The coincidence of diverse thermal artifacts is represented in Fig. 2.11, which shows a sample laser incision produced with a $\mathrm{CO}_{2}$ laser on ex-vivo chicken muscle tissue. The incision line was created through repeated scans of the laser beam: the high temperatures induced in correspondence of the laser incidence points determined the ablation of tissue. Carbonized tissue can be recognized in the surroundings of the incision crater, where the temperature of tissue has risen above $100{ }^{\circ} \mathrm{C}$. These areas appear darker in color with respect to normal tissue. Visual inspection of a broader area around the incision line

Table 2.2 Thermal effects of laser radiation on tissues. Reproduced from [5] with kind permission from Springer Science+Business Media

\begin{tabular}{l|l}
\hline Temperature $\left({ }^{\circ} \mathrm{C}\right)$ & Biological effect \\
\hline 37 & None \\
\hline 45 & Hyperthermia \\
\hline 50 & $\begin{array}{l}\text { Reduction in enzyme activity, cell } \\
\text { immobility }\end{array}$ \\
\hline 60 & $\begin{array}{l}\text { Denaturation of proteins and collagen, } \\
\text { coagulation }\end{array}$ \\
\hline 80 & Permeabilization of membranes \\
\hline 100 & Vaporization \\
\hline$>100$ & Carbonization \\
\hline$>300$ & Melting \\
\hline
\end{tabular}




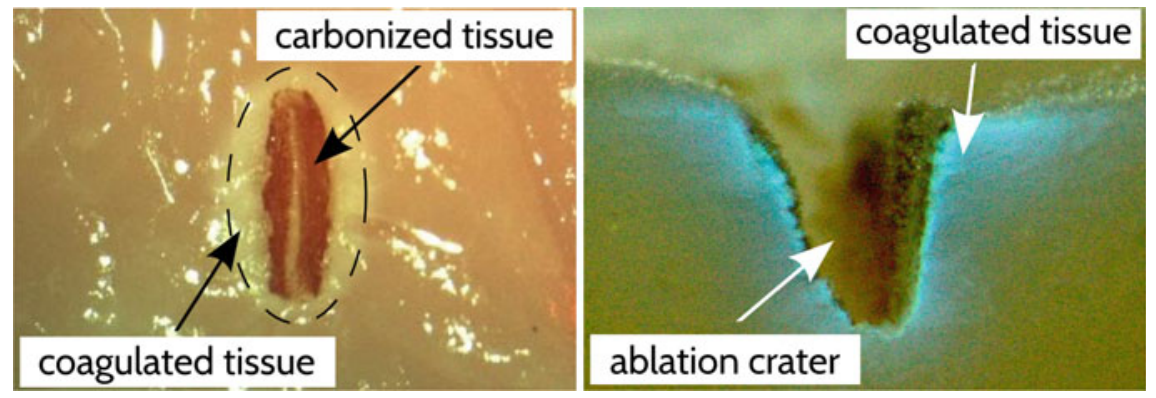

Fig. 2.11 Thermal effects of $\mathrm{CO}_{2}$ laser radiation on soft tissue. Here, the same tissue specimen is shown from a top view (left) and cross-section (right) perspective. Material ablation can be observed in correspondence with the laser incision line. Dark areas on the edges of the ablation crater indicate that temperatures over $100{ }^{\circ} \mathrm{C}$ have been reached, resulting in the carbonization of tissue. Coagulation can be also be observed, in the surroundings of the laser incision site.The volumetric extent of coagulation has been highlighted in the cross-section image through the use of a fluorescent light source

reveals the presence of coagulated tissue. A temperature below than $100{ }^{\circ} \mathrm{C}$ has been induced in these areas by means of heat conduction.

\subsubsection{Applications to Clinical Surgery}

Lasers are employed in a number of surgical specialties as cutting tools. Their use has gained popularity in those procedures where high accuracy is required, e.g. for the surgical treatment of glottic cancer [13]. With respect to traditional cutting tools, lasers present numerous advantages: they operate in a contact-less fashion, thus ensuring sterile conditions on the surgical site. In surgery of soft tissues, lasers can be tuned to simultaneously cut and coagulate blood, thus preventing excessive blood losses and limiting the need to resort to transfusions; this heamostatic effect further allows to keep the surgical site clean, enhancing the surgeon's assessment of the state of tissues [12, 14, 15]. Furthermore, lasers have enabled the development of new surgical techniques, such as the piece-wise tumor resection introduced by Steiner [14], which would have been impossible without a tool able to cut and coagulate surrounding tissues at the same time.

The knowledge of the absorbing and scattering properties of tissues is of paramount importance in laser surgery. Clinicians seek to create clean ablations with minimal thermal damage to surrounding tissues. The selection of a laser source with an appropriate wavelength is an essential prerequisite to achieve these goals. The $\mathrm{CO}_{2}$ laser is often indicated for surgery of soft tissues. These are rich in water, which is a strong absorber of the infrared radiation emitted by these class of lasers (wavelength: $10.6 \mu \mathrm{m}$ ). Alternatively, the solid-state Er:YAG laser (wavelength: $2940 \mathrm{~nm}$ ) is recommended for the ablation of bony tissue $[5,16]$. 
For surgical applications of lasers, carbonization of tissues surrounding the ablation site should be minimized as much as possible. It represents a non-intentional damage where healthy tissue that should have been preserved is being compromised, i.e. burned. Carbonization causes a longer recovery time for the patient and may leave scars, diminishing the quality of the procedure [12]. One common strategy to limit the extent of carbonization consists in the selection of laser exposure times shorter than the thermal relaxation time of tissue. In most soft tissues, laser irradiations shorter than $1 \mu \mathrm{s}$ do not produce significant thermal damage (this simple practical result is referred to as the "1 $\mu$ s rule") [5]. State-of-the-art $\mathrm{CO}_{2}$ surgical lasers present minimum pulse durations of a few hundred microseconds [13], thus potentially enabling thermal damage of surrounding tissues.

Laser surgery requires the dexterity to control the laser effects on tissues in order to obtain global appropriate results. These aspects will be discussed in detail in the next chapter. We shall see how the equipment currently in use for laser microsurgeries gives little support to clinicians to detect and understand the effects of laser actions.

Open Access This chapter is distributed under the terms of the Creative Commons AttributionNonCommercial 4.0 International License (http://creativecommons.org/licenses/by-nc/4.0/), which permits any noncommercial use, duplication, adaptation, distribution, and reproduction in any medium or format, as long as you give appropriate credit to the original author(s) and the source, a link is provided to the Creative Commons license, and any changes made are indicated.

The images or other third party material in this chapter are included in the work's Creative Commons license, unless indicated otherwise in the credit line; if such material is not included in the work's Creative Commons license and the respective action is not permitted by statutory regulation, users will need to obtain permission from the license holder to duplicate, adapt, or reproduce the material.

\section{References}

1. R. Haglund, The properties of light, ed. by F. Träger, in Springer Handbook of Lasers and Optics (Springer, New York, 2007), pp. 3-32

2. O. Svelto, S. Longhi, G. Valle, S. Kück, G. Huber, M. Pollnau, H. Hillmer, S. Hansmann, R. Engelbrecht, H. Brand, J. Kaiser, A. Peterson, R. Malz, S. Steinberg, G. Marowsky, U. Brinkmann, D. Lo, A. Borsutzky, H. Wähter, M. Sigrist, E. Saldin, E. Schneidmiller, M. Yurkov, K. Midorikawa, J. Hein, R. Sauerbrey, and J. Helmcke, Lasers and coherent light sources, ed. by F. Träger, in Springer Handbook of Lasers and Optics (Springer, New York, 2007), pp. 583-936

3. N. Lindlein, G. Leuchs, Wave optics, ed. by F. Trägerin, in Springer Handbook of Lasers and Optics (Springer, New York, 2007), pp. 87-156

4. W. Koechner, Optical resonator, in Solid-State Laser Engineering. Springer Series in Optical Sciences, vol. 1 (Springer New York, 2006), pp. 210-299

5. M. Niemz, Laser-tissue Interactions (Springer, Berlin, 2004)

6. M. Allmen, Laser-Beam Interactions with Materials: Physical Principles and Applications, Springer Series in Materials Science (Springer, Berlin, 2012)

7. D. Bäuerle, Laser Processing and Chemistry (Springer, Berlin, 2011) 
8. M. Brinkmann, J. Hayden, M. Letz, S. Reichel, C. Click, W. Mannstadt, B. Schreder, S. Wolff, S. Ritter, M. Davis, T. Bauer, H. Ren, Y.-H. Fan, S.-T. Wu, K. Bonrad, E. Krätzig, K. Buse, R. Paquin, Optical materials and their properties, ed. by F. Träger, in Springer Handbook of Lasers and Optics (Springer, New York, 2007), pp. 249-372

9. A. Vogel, V. Venugopalan, Mechanisms of pulsed laser ablation of biological tissues. Chem. Rev. 103(2), 577-644 (2003)

10. S.L. Jacques, Optical properties of biological tissues: a review. Phys. Med. Biol. 58(11), R37 (2013)

11. L. Fichera, D. Pardo, L. Mattos, Modeling tissue temperature dynamics during laser exposure, ed. by I. Rojas, G. Joya, J. Cabestany, in Advances in Computational Intelligence. Lecture Notes in Computer Science, vol. 7903 (Springer, Berlin, 2013), pp. 96-106. http://dx.doi.org/ 10.1007/978-3-642-38682-4_12 (Online)

12. R. Steiner, Laser-tissue interactions, in Laser and IPL Technology in Dermatology and Aesthetic Medicine, ed. by C. Raulin, S. Karsai (Springer, Berlin, 2011), pp. 23-36

13. M. Rubinstein, W. Armstrong, Transoral laser microsurgery for laryngeal cancer: a primer and review of laser dosimetry. Lasers Med. Sci. 26(1), 113-124 (2011)

14. W. Steiner, P. Ambrosch, Endoscopic laser surgery of the upper aerodigestive tract: with special emphasis on cancer surgery. Thieme (2000)

15. V. Oswal, M. Remacle, Transoral laser laryngeal surgery, in Principles and Practice of Lasers in Otorhinolaryngology and Head and Neck, 2nd edn., ed. by O. Vasant, R. Marc (Kugler Publications, Amsterdam, The Netherlands, 2014), pp. 99-116

16. C. Apel, R. Franzen, J. Meister, H. Sarrafzadegan, S. Thelen, N. Gutknecht, Influence of the pulse duration of an er: yag laser system on the ablation threshold of dental enamel. Lasers Med. Sci. 17(4), 253-257 (2002) 